\title{
Anti-Tracking in RFID Discovery Service for Dynamic Supply Chain Systems
}

\author{
Qiang Yan \\ Yingjiu Li \\ Robert H. Deng \\ School of Information Systems, Singapore Management University
}

\begin{abstract}
Unauthorized tracking of RFID tagged assets is one of the most important privacy concerns in RFIDenabled supply chain systems. Prior research has mainly focused on the prevention of unauthorized tracking by clandestine scanning at the physical level. However, the higher system level of unauthorized tracking has not been well recognized, where an adversary tracks movement of RFID tagged assets by eavesdropping network messages or compromising data center servers. Compared to unauthorized tracking at the physical level, unauthorized tracking at the system level could be even more harmful as the adversary is able to obtain tracking information on a global scale and without physical presence.

This paper analyzes the threat of unauthorized tracking by a semi-trusted RFID Discovery Service which maintains a database of RFID tag location records in the current industrial standard EPCglobal Network. We propose an anti-tracking design to mitigate this threat. Our design protects against reading attack to Discovery Service database and provides efficient key management and access control for dynamic supply chain systems. The design is backward compatible with the existing communication protocols as well as the database schemas of standard RFID Discovery Service, which makes it feasible to deploy in real world applications.
\end{abstract}

\section{Introduction}

With the advancement of information and communication technologies and cost reduction, RFID has been widely envisioned to be an inevitable replacement of barcodes and has been adopted as a new automatic identification technique for assets tracking in modern supply chain management systems. However, applications of RFID technique also dramatically increase the threat of illicit tracking of asset movement in RFID-enabled supply chain systems. Such asset movement information may disclose sensitive corporate information like secret co-operations or inventory strategies. Hence, prevention of unauthorized tracking of asset movement, or simply unauthorized tracking, constitutes one of the most imperative security and privacy issues in RFID-enabled supply chain management.

Unauthorized tracking may take place at physical level and/or system level in an RFID-enabled supply chain system. Prior research (e.g., [1][2][3]) has mainly focused on the prevention of clandestine scanning at physical level, where an adversary uses an unauthorized reader collecting RF waves to track the movement of RFID tags. The threat of unauthorized tracking at system level, where an adversary tracks movement of RFID tagged assets by eavesdropping network messages or compromising data center servers, however, has not been well recognized and studied in the literature. Compared to clandestine scanning, unauthorized tracking at the system level could be even more harmful as the adversary is able to obtain tracking information on a global scale and without physical presence.

To better understand unauthorized tracking at the system level, we analyze the threat of unauthorized tracking in the context of a semi-trusted RFID Discovery Service in the current industrial standard, EPCglobal Network [4]. RFID Discovery Service is one of the most important facility services in supply chain management. It is designed as a restrictedaccess search engine that provides RFID tag-level tracking information such as the locations of Information Services [5] associated with specific tag identifiers. In prior research [1][2][3][6][7][8][9], RFID Discovery Service is regarded as a trusted third party with security and privacy protection guaranteed by authentication and access control mechanisms. This same assumption is also adopted in industrial practices, where each supply chain partner is required to publish honestly its RFID tag location records, which contain the location of its Information Service and unique tag identifiers (e.g. Electronic Product Code [10]), to RFID Discovery Service, after it takes over the assets labeled with the tags.

However, placing total trust on RFID Discovery Service is problematic and violates the basic security principle of least privilege. First, there is no single 
organization universally trusted by all companies to protect their sensitive corporate information. Second, even if such trusted third party organization exists, compromise of Discovery Service, by either outside hackers or inside attackers (e.g., incompetent or corrupt employees), may expose all asset movement information all supply chains serviced by Discovery Service. This vulnerability is likely to be exploited especially when Discovery Services are deployed as Internet services, as recommended by the latest EPCglobal specifications [10]. This threat was also identified in [8], where it was suggested that all client organizations choose RFID Discovery Services they trust.

To mitigate the above privacy threat, protection against such unauthorized tracking should be considered in the design of RFID Discovery Service. In this paper, we propose an anti-tracking design for a semi-trusted RFID Discovery Service which has the following advantages:

- Protection against database reading attack. Our design prevents RFID Discovery Service from learning tracking information from RFID tag location records maintained in its database. The information that can be used to link any two location records associated with the same tag is concealed using pseudonyms and timestamp encryption.

- Efficient key management and access control. We assign independent secret keys (for pseudonym generation and timestamp encryption) to supply chain partners in order to satisfy advanced and dynamic traceability requirements. Our design allows for efficient key management and privilege revocation even for dynamic supply chain systems.

- Backward compatibility. The design is backward compatible with existing communication protocols and database schemas of RFID Discovery Service, and it does not impose any extra requirements on the physical RFID infrastructure.

The rest of the paper is organized as follows. We summarize the related work in Section 2. In Section 3, we review the RFID Discovery Service architecture and introduce our threat model. Sections 4,5 , and 6 are the main contribution of this paper, where we present anti-tracking design (section 4), key management and access control (section 5) and compatibility and performance analysis (section 6) in detail. We discuss several practical considerations in Section 7 and finally conclude the paper in Section 8.

\section{Related Work}

Unauthorized tracking, as one of the major privacy issues in RFID systems, has been extensively investigated in recent years. Prior research has focused on the threat of unauthorized tracking caused by clandestine scanning at physical level. Many communication protocols are proposed to address this problem. A few representative works includes: Weis et al. [1] proposed the hash lock solution that uses a one-way hash function to lock a tag. A tag always responds a random number unless a correct key is provided to unlock the tag. Henrici and Müller [2] changed the state value stored in the tag for response generation after each successful authentication to avoid tracking on the same response. Jues et al. [3] proposed to use a more powerful trusted agent called RFID Enhancer Proxy to periodically re-label the identifiers of the low-cost tags and simulate spurious tags to hide the physical tracking information. The protocol design becomes more challenge for large-scale industrial RFID systems which requires the extra supports such as ownership transfer and third party logistics. To support these features, a protocol should allow a party to access necessary contents stored in tags without the full knowledge of the secret key. And this access privilege is subject to change. More details about these protocol designs can be found in a recent survey by Langheinrich [11].

There are a few works that also identified the threat of unauthorized tracking at system level. Fabian et al. [6] provided a security analysis for the object name service [10], which is a public instance of RFID Discovery Services. They suggested using the relay-based anonymity preserving techniques to mitigate the threat of unauthorized tracking for the location of user who queries the object name service. However, these techniques will not protect user's identity as it is usually revealed to enforce access control on RFID Discovery Services. Burbridge and Harrison [8] recommended that all client organizations be allowed to choose RFID Discovery Services they trust. None of the previous works has attempted to resolve this issue by enhancing the design of RFID Discovery Services in a directory service model as what we propose in this paper.

\section{RFID Discovery Service and Threat Model}

\subsection{RFID Discovery Service and Its Vulnerability}

A typical application scenario using RFID Discovery Service in an RFID-enabled supply chain system consists of the following three types of entities:

- RFID Discovery Service: a restricted-access search engine that functions as a depository of RFID tag location records and performs access control for authorized users to access the records. 
- Supply chain partners who maintain warehouses and transport RFID tagged physical assets from one location to another location and provide detailed supply chain information from their respective Information Services.

- Authorized users who have the privileges to access the location records by querying RFID Discovery Service. An authorized user could be a supply chain partner.

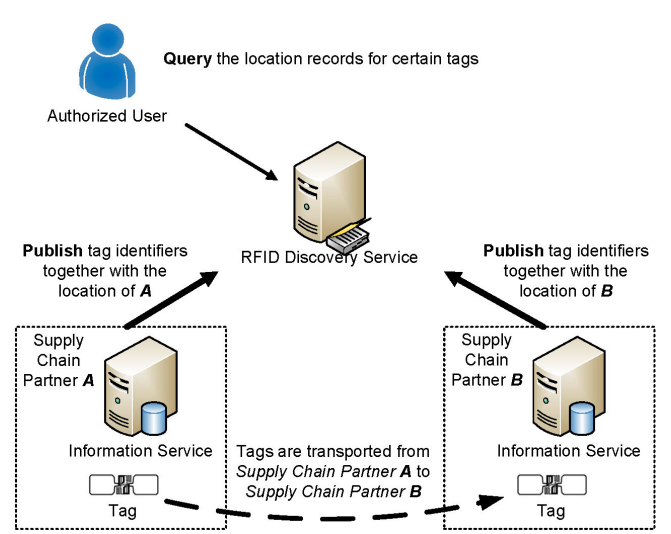

Figure 1. Application scenario of RFID Discovery Service

Figure 1 illustrates the interaction among these three types of entities. Since RFID Discovery Service is regarded as a fully trusted third party in EPCglobal Network, a supply chain partner publishes plaintext RFID tag location records to RFID Discovery Service after it takes over the assets labeled with those tags. A tag location record follows the format of $\langle T I D, L O C A T I O N, T I M E\rangle$, where TID is a tag identifier such as the Electronic Product Code (EPC code), LOCATION is a pointer to the supply chain partner's Information Service, and TIME is the time of the record creation. A RFID tag's or equivalently a RFID tagged asset's tracking information in a supply chain system is the locationtime sequence embedded in the location records associated with the tag. Such information indicates that the tag arrived at which supply chain partner at what time. To track the movement of a specific tag, an authorized user, upon authentication, may retrieve all the location records indexed by the tag identifier, and then obtain further information regarding the tag (or the asset associated with the tag) from the Information Services pointed to by LOCATIONs.

Obviously, RFID Discovery Service has access to all location records maintained in its database and therefore is able to track the movement of any tags in the supply chain system. Figure 2 shows a simple example of Discovery Service tracking tag movement through tag identifier grouping. RFID Discovery Service knows that a tag with a unique identifier EPCl was transported from supply chain partner A to supply chain partner B by grouping the location records indexed by EPC1, $\angle E P C 1, L 1, \ldots>$ and $\langle E P C 1, L 2, \ldots\rangle$, where $L 1$ and $L 2$ are the locations of the Information Services maintained by supply chain partners $A$ and $B$, respectively.

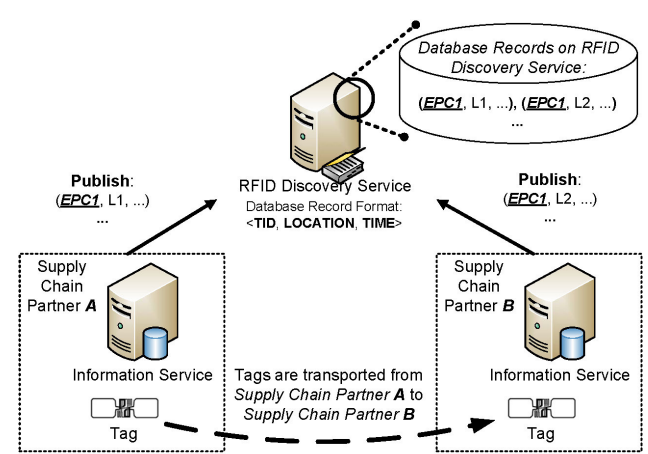

Figure 2. Example of movement tracking by RFID Discovery Service through tag identifier grouping

RFID Discovery Service can also infer tag movement information from location records by grouping and correlating timestamps, even if tag identifiers are concealed using random-looking pseudonyms, as demonstrated in Figure 3. Discovery Service can deduce that a batch of two tags with pseudonyms $P 1, P 2, P 3$, and $P 4$ may have been transported from supply chain partner $A$ to supply chain partner $B$, by grouping and correlating timestamps $T 1$ and $T 2$ in the location records $\angle P 1$, $L 1, T 1>,<P 2, L 1, T 1>,<P 3, L 2, T 2>$, and $<P 4, L 2$, $T 2>$.

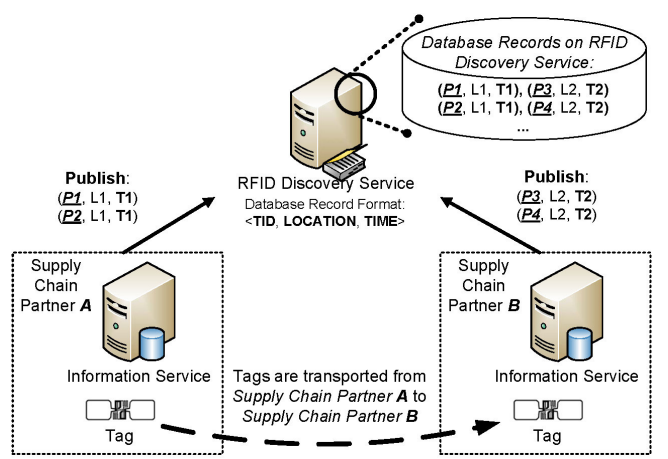

Figure 3. Example of movement tracking by RFID Discovery Service through grouping and correlating of timestamps

The current RFID Discovery Service specifications by EPCglobal Network are undoubtedly not resilient against tag tracking attacks initiated at the Discovery Service side. Compromise of Discovery Service by either outsiders or insiders subjects all the tags'/assets' movement information to exposure and may have serious legal and financial repercussions to supply chain partners as well as to the organization who operates Discovery Service. 


\subsection{Threat Model}

The adversaries in our threat model include RFID Discovery Service and other outliers. The capabilities of these adversaries are defined as follows:

- RFID Discovery Service is semi-trusted. Specifically, RFID Discovery Service is controlled by a passive adversary which follows honest-but-curious behavior. That is, Discovery Service honestly executes operations according to its system specifications - authenticates users and supply chain partners, maintains the integrity of all the location records in its database, and answers users' queries faithfully. However, Discovery Service is curious in the sense that it desires to infer tags' movement information by mining location records in its database. Since RFID Discovery Service is usually an outsourcing service maintained by an organization, it is necessary for the organization to faithfully follow the system's operational specifications in order to run the business and without its malicious intentions being detected.

- Outliers are passive attacker. They try to track tags' movements by eavesdropping network messages.

The other entities in our threat model are supply chain partners and authorized users.

- All supply chain partners and authorized users are assumed to be trusted so that they do not disclose secret keys or collude with the adversaries.

We assume that all message exchanges between users/supply chain partners and RFID Discovery Service are carried over confidentiality and integrity protected channels. Note that our design is for dynamic supply chain systems. So there is no prerequisite for a certain static structure among supply chain partners and authorized users. Any supply chain partners and authorized users may join or leave the system anytime.

This threat model is stronger than the traditional threat models used in studying unauthorized tracking problems of RFID systems [1][6][8][9]. In our threat model, RFID Discovery Service is an adversary. Since Discovery Service is always able to read the database whether transport layer security mechanism is used or not, it is more powerful than the outliers which are only able to eavesdrop network messages. Hence, it suffices to consider RFID Discovery Service as the sole adversary in the following discussion.

\section{Anti-Tracking Design in Discovery Service}

Our objective in designing a privacy-preserving RFID Discovery Service is to minimize the amount of tracking information learned by RFID Discovery Service, so that disclosure of such information will be minimized even when RFID Discovery Service is compromised. Besides the privacy concerns, we still need to provide efficient mechanisms for authorized users to obtain tag level tracking information by querying Discovery Service. The design challenge therefore is to balance the tradeoff between privacy and performance. It is also important to maintain backward compatibility for practical deployment.

\subsection{Unauthorized Tracking Mitigation}

The key idea to mitigate tracking information disclosure from database reading is hiding sensitive fields in the location records to prevent grouping and correlating location records associated to the same underlying RFID tag. As we have demonstrated in Figure 2 and Figure 3, such sensitive fields are the tag identifier and the timestamp. However, such fields cannot be simply distorted or suppressed as business value of location records drops dramatically when the precise values of these fields are made inaccessible [8]. To preserve the precise information while hiding sensitive fields from database reading attack, we replace tag identifiers with pseudonyms and encrypt timestamps.

1) Pseudonym Indexing: We employ pseudonyms instead of the original tag identifiers to index location records stored in the database of RFID Discovery Service. Unlike the original tag identifiers which are invariant throughout the lifetime of a supply chain system, pseudonyms generated by different supply chain partners, though corresponding to the same tag identifier, appear differently. We use a keyed pseudorandom function $F(.,$.$) , such as the keyed Hash Message$ Authentication Code (HMAC), to generate pseudonyms, Pseudonym $=F($ secret $k e y$, original tag identifier). Such pseudonyms possess the following desirable properties:

- $\quad$ The mapping from an original tag identifier and a secret key to a pseudonym is deterministic. Otherwise authorized users will not be able to re-generate the same pseudonyms in order to retrieve location records from Discovery Service.

- It is computationally infeasible to link a pseudonym to its underlying original tag identifier without knowing the secret key. This means that the adversary cannot link any two location records indexed by two different 
pseudonyms generated from the same tag identifier.

Pseudonyms are generated by supply chain partners and used in place of the original tag identifiers when publishing location records to Discovery Service. Each supply chain partner is assigned a different secret key; otherwise two different supply chain partners will generate the same pseudonym for the same original tag identifier, which will defeat the purpose of using multiple unlinkable pseudonyms to break the correlation among the location records associated with the same original tag identifier.

2) Timestamp Encryption: Disclosure of tracking information from timestamps is orthogonal to that from tag identifiers. The adversary may link multiple location records by grouping and correlating a series of time stamps regardless of pseudonym indexing is used or not (see Figure 3).

To mitigate this attack, we use encrypted timestamps, instead of plaintext timestamps, in location records. Note that timestamp encryption does not affect the semantics of RFID Discovery Service as the time of record creation is not an index field used in the queries to RFID Discovery Service. The encryption algorithm for timestamp encryption must be probabilistic. Specifically we use a $C P A$ secure symmetric encryption scheme $E(.,$.$) , such as$ AES in CBC mode with random initialization vector (AES-CBC). A CPA-secure encryption scheme introduces randomness in the ciphertext so that the same plaintext timestamp will not produce the same ciphertext when encrypted twice using the same key. As a result, the adversary will not be able to link any two location records associated with the same time event but labeled by different ciphertexts of the same timestamp.

It is generally recognized as a prudent practice to use different secret keys for different cryptographic operations. In our case, a supply chain partner uses one secret key for pseudonym generation and another secret key for timestamp encryption. To simplify the discussion however, we assume that a supply chain partner uses one secret key for both pseudonym generation and timestamp encryption.

\subsection{Revised Operation Model}

For easy of reference, Table 1 summarizes the common notations used during the rest of the paper.

\section{Table 1. Common notations}

\section{For a supply chain partner $p$ :}

$k_{p}$ is the secret key for pseudonym generation and timestamp encryption.

$l o c_{p}$ is the location of its Information Service.

For a $\operatorname{tag} x$ : $t_{x}$ is the tag identifier.

$p n_{x, p}$ is the pseudonym generated by supply chain partner $p$.

$e t_{x, p}$ is the ciphertext of timestamp $T_{p}$ generated by supply chain partner $p$.

\section{Other notations:}

$F(k, m)$ is the pseudorandom function with secret key $k$ and message $m$ as input.

$E(k, m)$ is the encryption of message $m$ using secret key $k$.

The operational model of our proposed RFID Discovery Service consists of six basic operations.

- Publish $(p, x)$ : a supply chain partner $p$ publishes a location record for a tag $x$.

- $\quad \operatorname{Query}(P, x)$ : an authorized user retrieves all location records of a tag $x$ published by a subset $P$ of all supply chain partners in a supply chain.

- $\operatorname{Join}(p)$ : a new partner $p$ joins the supply chain.

- Leave $(p)$ : an existing partner $p$ leaves the supply chain.

- $\operatorname{Grant}(u)$ : grant privilege of accessing certain location records to a user $u$.

- Revoke $(u)$ : revoke user $u$ 's privilege of accessing certain location records.

The first two basic operations (shown below) provide the essential functionality of tag level tracking.

\section{$\operatorname{Publish}(p, x)$ :}

Supply chain partner $p$ :

1. Compute $p n_{x, p}=F\left(k_{p}, t_{x}\right)$

2. Compute $e t_{x, p}=E\left(k_{p}, T_{p}\right)$

3. Publish a location record $\left\langle p n_{x, p}, \quad l o c_{p}, e t_{x, p}\right\rangle$ to RFID Discovery Service.

$\operatorname{Query}(P, x)$ :

An authorized user $u$ with privilege $\operatorname{Pr} \lg (u)$ :

1. Set $P N=\varnothing$

2. For each supply chain partner $p \in P$ and $k_{p} \in$ $\operatorname{Prlg}(u)$

Compute $p n_{x, p}=F\left(k_{p}, t_{x}\right), P N=P N \cup p n_{x, p}$

3. Send $P N$ to RFID Discovery Service.

RFID Discovery Service stores location records $<p n_{x, p}, \quad l o c_{p}, e t_{x, p}>$ in its database. Note that the location information $l o c_{p}$ is not encrypted as it will be used to enforce access control on RFID Discovery Service (see Section 5.2).

To retrieve all location records associated with a tag identifier $t_{x}$, an authorized user must be able to regenerate the pseudonyms in these location records; in other words, the authorized user must know the secret keys of the supply chain partners who published these records in the first place. Hence, a user $u$ 's privilege $\operatorname{Prlg}(u)$ contains at least the secret 
keys of supply chain partners whose location records are allowed access by user $u$. Management of user privileges is facilitated via the last four basic operations, which will be described in detail in the next section.

Referring to the Publish $(p, x)$ operation, upon reception of user $u$ 's query $Q u e r y(P, x)$, RFID Discovery Service replies with all the location records $\left\{<p n_{x, p}, l o c_{p}, e t_{x, p}>\right\}$ that are indexed by $\left\{p n_{x, p} \mid p n_{x, p} \in P N\right\}$. The user then decrypts $e t_{x, p} \mathrm{~s}$ to get the plaintext timestamps and therefore the location-time sequence for $\operatorname{tag} x$.

\section{Key Management and Access Control}

The challenge of key management and access control stems from the functional restrictions of semi-trusted RFID Discovery Service. Specifically, to prevent disclosure of tracking information to Discovery Service, it is required that the secret keys used in pseudonym generation and timestamp encryption not be disclosed to Discovery Service. Another challenge is to efficiently handle user revocations. This section progressively presents our solutions for key management and access control for dynamic supply chain systems.

\subsection{Scheme I: Supporting flexible tag level tracking}

Modern supply chain systems require flexible tag level tracking where a user may be authorized to access any location record published by any supply chain partner. The basic idea to satisfy this generic requirement is using independent key assignment. Unlike hierarchical key generation schemes [12] which assume users' privileges following certain static hierarchical structures, independent key assignment is more flexible for dynamic supply chain systems. With independent key assignment, each supply chain partner $p$ is assigned an independent random number as its secret key $k_{p}$ for pseudonym generation and timestamp encryption, for $p=1,2, \ldots, N$, where $N$ is the number of partners in the supply chain. Supply chain partners publish their location records to RFID Discovery Service using the operation Publish $(p, x)$ given in the previous section.

A user $u$ queries Discovery Service via the operation $\operatorname{Query}(P, x)$. To query location records published by supply chain partner $p \in P$, the user is given supply chain partner $p$ 's secret key $k_{p}$. Therefore, user $u$ 's access privilege $\operatorname{Pr} \lg (u)$ is the set of secret keys of those supply chain partners whose location records are allowed access by $u$.

In a dynamic supply chain system, partners may join and leave the system anytime. As a result, privileges of all authorized users need to be updated and disseminated to users in a dynamic and timely manner. For this purpose, we introduce an online security manager for managing users' privileges. Naturally, the online security manager has the highest privilege and must be trusted by all the partners in a supply chain system. We remark that the online security manager is supply chain specific while Discovery Service serves multiple supply chains across the Internet. Though it may be difficult or impossible to find a fully trusted Discovery Service, it is highly reasonable to assume the existence of a fully trusted online security manager. For instance, the online security manager could be operated by the dominant partner which usually exists in a supply chain system (e.g., a well-known manufacturer or retailer, such as Wal-Mart [13]).

The basic operations of the online security manager are given as follows.

\section{$\operatorname{Join}_{S I}(p)$}

Generate secret key $k_{p}$ for a newly joined supply chain partner $p$ and update privileges of all affected users.

Leave $_{S 1}(p)$ :

No action is required upon departure of an existing partner $p$.

$\operatorname{Prlg}(u)=\left\{k_{p} \mid k_{p}\right.$ is the secret key of supply chain partner $p \in P$, where $P$ is the set of supply chain partners whose location records are allowed access by user $u$.

\section{$\operatorname{Grant}_{S I}(u)$ :}

Grant privilege $\operatorname{Prlg}(u)$ to user $u$ upon user authentication.

Before querying Discovery Service, a user $u$ first acquires his/her privilege $\operatorname{Prlg}(u)$ from the online security manager. This ensures that the user's privilege is not out-of-dated. It is assumed that all communications between the online security manager and the users take place over secure channels.

\subsection{Scheme II: Supporting user revocation}

Scheme I does not handle user revocation and hence is only suitable for key distribution and access control for supply chain systems with static user groups.

In Scheme I, a user $u$ is granted an access privilege $\operatorname{Prlg}(u)=\left\{k_{p} \mid k_{p}\right.$ is the secret key of supply chain partner $p \in P\}$. Since the secret keys of supply chain partners are permanent in Scheme I, user $u$ 's privilege $\operatorname{Prlg}(u)$ is permanent too. That is, user $u$ can access supply chain partner $p$ 's location records forever even after the user has long left the system, which is undesirable. Once a user leaves the system or is revoked, the user must be prevented from accessing future location records in the supply chain system. 
One straightforward approach to handle user revocation is assigning new secret keys to supply chain partners whenever a user is revoked. Supply chain partners then erase their previous secret keys and use the new secret keys for pseudonym generation and timestamp encryption when publishing records. However, it will be impractical to assign new secret keys to supply chain partners when the user group is large or user revocation is frequent. A more feasible method could be to divide time into intervals of fixed duration, say 24 hours, and all supply chain partners refresh their secret key values at the beginning of each interval. Unfortunately, the performance cost is still high even with a controlled (probably reduced) frequency of key update. In addition to the high cost of key management, periodical secret key update significantly reduces query efficiency as a user has to enumerate all the secret keys and regenerate all possible pseudonyms to retrieve the location records of a tag. Specifically, assume user $u$ want to retrieve the location record of tag $t_{x}$ published by partner $p$. Instead of computing just one pseudonym as in Scheme I, the user has to compute and submit $n$ pseudonyms, $F\left(k_{p, i}, t_{x}\right)$, for $i=$ $1,2, \ldots, n$, where $k_{p, i}$ is the secret key assigned to partner $p$ at time interval $i$ and $n$ is the number of time internals elapsed since the start of the supply chain. The computation complexity of querying one location record is thus $O(n)$.

In order to efficiently support user revocation, Scheme II is designed to minimize the cost of key update, where Discovery Service implements a finergrained user access control to prevent revoked users from retrieving location records. To this end, we slightly extend database schemas to include certain auxiliary information which Discovery Service rely upon to enforce access control. The auxiliary fields should be carefully selected to limit disclosure of tracking information. We can use coarse-grained time, say a day, as the auxiliary field. The basic operations of the online security manager are given as follows. A user $u$ 's privilege is the same as in Scheme I, i.e., given by $\operatorname{Pr} \lg (u)=\left\{k_{p} \mid k_{p}, p \in P\right\}$. The access control policy for user $u$ is given by $\operatorname{Policy}(u)=\left\{<u\right.$, loc $_{p}$, day d $_{i}$, permission $\left.>\mid p \in P\right\}$, where $P$ is the set of supply chain partners whose location records are allowed or denied access by user $u$.

\footnotetext{
$\operatorname{Join}_{S 2}(p)$ :

1. Generate secret key $k_{p}$ for supply chain partner $p$.

2. Update user privilege $\operatorname{Prlg}(u)$ for all affected users.

3. Update access control policy Policy $(u)$ for all affected users and forward them to RFID Discovery Service.

Leave $_{S 2}(p)$ :

1. Update user privilege $\operatorname{Prlg}(u)$ for all affected users.
}

2. Update access control policy Policy $(u)$ for all affected users and forward them to RFID Discovery Service.

$\boldsymbol{G r a n t}_{S 2}(u)$ :

Grant privilege $\operatorname{Prlg}(u)$ to user $u$ upon user authentication.

Revoke $_{S 2}(u)$ :

Instruct Discovery Service deleting Policy $(u)$ for user $u$.

The query process is almost the same as in Scheme I, where a user $u$ first acquires $\operatorname{Pr} \lg (u)$ from the online security manager, and then generates pseudonyms to retrieve the required location records. So the security manager in Scheme II still has to be online for the same reason as in Scheme I. The difference is that RFID Discovery Service now prevents revoked users from accessing its database based on enforced access control policies.

\subsection{Scheme III: Supporting user revocation without online security manager}

Both Schemes I and II require the existence of an online security manager. An online security manager increases the operational cost and is cumbersome to use for users. To eliminate the requirement of an online security manager, Scheme III employs proxy re-encryption scheme [14] to shift the burden of the online security manager to RFID Discovery Service.

Proxy re-encryption is a technique that allows a proxy to transform a ciphertext computed under Alice's public key into one that can be decrypted using Bob's private key. During ciphertext transformation, referred to as re-encryption, the proxy learns nothing about the underlying plaintext. A proxy re-encryption scheme is represented as a tuple of (possibly probabilistic) polynomial time algorithms $(K G, R G, E, R, D)[15]$ :

- $(K G, E, D)$ are the standard key generation, encryption, and decryption algorithms for an underlying public key encryption scheme. On input the security parameter $1^{k}, K G$ outputs a public and private key pair $\left(p k_{A}, s k_{A}\right)$. On input $p k_{A}$ and message $m, E$ outputs a ciphertext $C_{A}=E\left(p k_{A}, m\right)$. On input $s k_{A}$ and ciphertext $C_{A}, D$ outputs the message $m=$ $D\left(s k_{A}, C_{A}\right)$.

- On input $\left(p k_{A}, s k_{A}, p k_{B}\right)$, the re-encryption key generation algorithm, $R G$, outputs a reencryption key $r k_{A \rightarrow B}$ for the proxy.

- On input $r k_{A \rightarrow B}$ and ciphertext $C_{A}$, the reencryption function, $R$, outputs $R\left(r k_{A \rightarrow B}, C_{A}\right)$ $=E\left(p k_{B}, m\right)=C_{B}$ which can be decrypted using private key $s k_{B}$.

In Scheme III, the offline security manager as well as every user each has a public and private key pair under the public key encryption scheme $(K G, E$, 
$D)$. Let $\left(p k_{S M}, s k_{S M}\right)$ denotes the public and private key pair of the offline security manager and $\left(p k_{u}, s k_{u}\right)$ denote the public and private key pair of user $u$. On a high level, Scheme III works as follows. The offline security manager, on input $\left(p k_{S M}, s k_{S M}, p k_{u}\right)$ to the algorithm $R G$, generates the re-encryption key $r k_{S M \rightarrow u}$ for every user $u$. The offline security manager then forwards all $r k_{\mathrm{SM} \rightarrow \mathrm{u}}$ 's and users' access control policies to RFID Discovery Service. Supply chain partner $p$ encrypts its secret key $k_{p}$ using the public key of the offline security manager to obtain $E\left(p k_{S M}\right.$, $k_{p}$ ) and publishes it along with its location records to Discovery Service. If user $u$ is allowed to access supply chain partner $p$ 's location records, Discovery Service computes $R\left(r k_{S M \rightarrow u}, E\left(p k_{S M}, k_{p}\right)\right)=E\left(p k_{u}, k_{p}\right)$ and gives it to user $u$. The user decrypts $E\left(p k_{u}, k_{p}\right)$ using his/her private key $s k_{u}$ to obtain $k_{p}$ and uses it to query the database. In Scheme III, Discovery Service functions as the proxy in the proxy reencryption scheme, it indirectly distributes secret keys of supply chain partners to users while without learns anything about these secrets. Basic operations of Scheme III are presented as follows.

\section{$\operatorname{Join}_{S 3}(p)$ :}

Supply chain partner $p$ :

1. Generate a random secret key $k_{p}$.

2. Acquire the public key $p k_{S M}$ of the offline security manager.

3. Compute $E\left(p k_{S M}, k_{p}\right)$, and send it to RFID Discovery Service.

Offline security manager:

4. Update access control policy Policy $(u)$ for all affected users and forward them to RFID Discovery Service.

Leave $_{S 3}(p)$ :

Offline security manager updates access control policy Policy $(u)$ for all affected users and forwards them to RFID Discovery Service.

$\operatorname{Policy}(u)=\left\{\left\langle u\right.\right.$, loc $_{p}$, day $_{i}$, permission $\left.\left.>\right| p \in P\right\}$, where $P$ is the set of supply chain partners whose location records are allowed or denied access by user $u$.

\section{$\boldsymbol{G r a n t}_{S 3}(u)$ :}

1. User $u$ generates $\left(p k_{u}, s k_{u}\right)$ : $\left(p k_{u}, s k_{u}\right)=K G\left(1^{k}\right)$

2. Offline security manager generates $r k_{S M \rightarrow u}$ : $r k_{S M \rightarrow u}=R G\left(p k_{S M}, s k_{S M}, p k_{u}\right)$

3. Offline security manager sends $r k_{S M \rightarrow u}$ and Policy $(u)$ to RFID Discovery Service.

Note: Steps 1, 2, and 3 are skipped if they have been executed before and the values of $\left(p k_{u}, s k_{u}\right)$ and $\left(p k_{S M}, s k_{S M}\right)$ remain unchanged.

4. User $u$ requests transformed ciphertext of the secret key $k_{p}$ of supply chain partner $p$ from RFID Discovery Service.
5. RFID Discovery Service checks Policy $(u)$ and transforms $E\left(p k_{S M}, k_{p}\right)$ into $E\left(p k_{u}, k_{p}\right)$ if Policy $(u)$ authorizes $u$ to access $p$ 's location records:

$$
R\left(r k_{S M \rightarrow u}, E\left(p k_{S M}, k_{p}\right)\right)=E\left(p k_{u}, k_{p}\right)
$$

6. User $u$ obtains $k_{p}$ by decrypting $E\left(p k_{u}, k_{p}\right)$ using his/her private key $s k_{u}$ :

$$
k_{p}=D\left(s k_{u}, E\left(p k_{u}, k_{p}\right)\right) \text {. }
$$

\section{Revoke $_{S 3}(u)$ :}

Offline security manager removes Policy $(u)$ from RFID Discovery Service.

Compared to Schemes I and II, Scheme III replaces the online security manager with an offline security manager. The security manager can now stay offline unless access control policies change when a user or supply chain partner join or leave the supply chain system. Scheme III also introduces a pair of public and private keys for the offline security manager and for each user. It requires authenticated distribution of a) the public key of the offline security manager to all users, b) public keys of all users to the offline security manager, and c) all reencryption keys from the offline security manager to Discovery Service. In most public key cryptosystems, a significant portion of the operational cost is attributed to management of public keys (e.g., authenticated distribution and revocation of public keys). Fortunately, the management of public keys in our system is simple and can be done efficiently, as it is actually a prerequisite. There should be existing authenticated and secure channels between the offline security manager, Discovery Service and users, even without the use of public key encryption introduced in our scheme. Hence, the overhead due to the management of public keys in Scheme III is not significant.

\section{Compatibility and Performance Analysis}

Below we summarize the modifications to standard EPCglobal Discovery Service by adopting our enhanced design.

- Query users: Users are required to acquire secret keys of supply chain partners before querying location records. To retrieve all the location records corresponding to a tag identifier, a user now needs to issue a set of pseudonyms instead of using the original tag identifier as the query parameter. In Scheme III, a user needs to generate a pair of public keys and use proxy re-encryption for obtaining those secret keys.

- Supply chain partners: Secret keys are to be generated for pseudonym indexing and timestamp encryption during publishing the location records. The secret keys are also to 
be encrypted and updated to Discovery Service in Scheme III.

- RFID Discovery Services: The data structures of our records are compatible with the existing ones. Only two semantic changes are required: one is the original tag identifier replaced with a pseudonym; the other is the plaintext timestamp replaced with encrypted timestamp. Auxiliary fields may be needed to enforce fine-grained access control. For Scheme III, Discovery Service would need to do ciphertext transformation.

- Security managers: An online security manager is needed in Scheme I and II, while an offline security manager is required in Scheme III for generating re-encryption keys and managing and updating access control policies as well.

As shown above, only minor changes are required to adopt our design. With unaltered physical RFID infrastructure, both trusted and semi-trusted RFID Discovery Services can be practically designed into one serving system which provides seamless RFID Discovery Services at different security levels.

With enhanced privacy protection, our antitracking design inevitably impacts the performance of RFID Discovery Service as it introduces extra cryptographic operations. We estimate this impact by implementing a demo system of Scheme III based on the JHU-MIT Proxy Re-cryptography Library [16]. The demo system is implemented in $\mathrm{C}++$ on Ubuntu Linux. We choose the security strength of 128-bit AES in our demo system. Thus we use HMAC with SHA-256 for pseudonym generation, AES-CBC with 128-bit key for timestamp encryption, and bilinear El Gamal proxy re-encryption scheme with 256-bit key [15] for proxy re-encryption. Our experimental machine consists of a $2.53 \mathrm{GHz}$ Intel Core 2 Duo E7200 processor and 4GB DDR2 800Hz RAM.

In our experiments, on average, it takes $0.30 \mu$ s to generate a pseudonym (3.28M pseudonyms/sec), and $0.16 \mu$ s to encrypt a timestamp $(6.25 \mathrm{M}$ timestamps/sec). For proxy re-encryption, it takes $3.3 \mathrm{~ms}$ to encrypt a secret key, and $8.6 \mathrm{~ms}$ to reencrypt the ciphertext of a secret key, and $1.6 \mathrm{~ms}$ to decrypt the ciphertext after re-encryption. Although the computation of re-encryption scheme is much slower than HMAC and AES-CBC, its performance cost would not become the performance bottleneck. It is because the results of re-encryption can always be reused as long as the ciphertexts of secret keys remain unchanged. Hence, the overall additional cost is moderate after adopting our enhanced design.

\section{Practical Considerations}

\subsection{Mitigating Disclosure of Tracking Information from Access Patterns}

Access patterns are the sequence of user queries and database answers as well as the sequence of timestamps of publishing location records. Through observing access patterns, the adversary may be able to infer the correlation among a group of location records when they are referred to in the same query or published at the same time. It is difficult to completely prevent the adversary from learning tracking information based access patterns without employing complex and sometimes impractical techniques such as Private Information Retrieval (PIR) [17]. However, we believe that there are practical methods which can partially mitigate the disclosure of tracking information from access patterns, such as splitting related pseudonyms into separate queries, or introduce delays or dummy data in publishing location records to Discovery Service. We will investigate the effectiveness of such methods in our next step of research.

\subsection{Mitigating Disclosure of Tracking Information from Collusion Attack}

Collusion could happen among users, supply chain partners and RFID Discovery Service. The best effort against collusion attack is that the tracking information disclosed to the accomplices should not be more than the tracking information that they have already known. Hence the threat from collusion involving a user and a supply chain partner is not a serious issue as no more tracking information is disclosed to accomplices than that they already know.

However, collusion attacks involving RFID Discovery Service is a really serious threat. Consider the following scenario. A user $u$ with knowledge of $\operatorname{Prlg}(u)=\left\{k_{p} \mid k_{p}\right.$ is the secret key of supply chain partner $p \in P\}$ is revoked. The user is therefore denied future access to location records published by supply chain partner $p \in P$ due to access control at Discovery Service. However, if the user colludes with Discovery Service, together they are able to access any location records published by $p \in P$. Updating secret keys of supply chain partners upon user revocation is a remedy to this kind of collusion attacks. But, as we have discussed in Scheme II, frequent secret key update significantly degrades query efficiency and increases the complex of key management. Another approach to counter collusion attacks is to employ tamper-resistant hardware such as the Trusted Platform Module (TPM) in the Trusted Computing Platform technology. Secret keys are stored inside the tamper-resistant TPM and all 
cryptographic operations involving the secret keys, such as pseudonym generation and timestamp encryption, take place inside the TPM. The accomplices would have to stay online to perform collusion with RFID Discovery Service, which makes them to be detected more easily.

\subsection{Enhancing Privacy Protection for Supply Chain Partners with Small Product Category}

Different from large supply chain partners, small supply chain partners only have a small product category associated with them. For example, a small supply chain partner may be responsible to transport coal only so that all tracking information associated with this supply chain partner is about coal. If this strict association information is available to an adversary who has compromised an RFID discovery service, it is easy to identify related tracking information by reading the plaintext location information in the database. Encryption on location information alone is not able to prevent the adversary from learning the plaintext location information by the disclosed identity of supply chain partner who publish this location information during the authentication process.

One way to enhance privacy protection for this kind of supply chain partners is to group them with other supply chain partners to form anonymity groups. An anonymity group contains a sufficient number of supply chain partners so that the association between products and the anonymity group is not strict. Each request for location update from an anonymity group should be relayed by a proxy and use the anonymous credential [18] in the authentication so that only the identity of the anonymity group is disclosed to RFID discovery service. A proxy could be a relay-based anonymity network like TOR [19]. The location information in these requests is also required to be encrypted otherwise RFID discovery service is still able to tell the true identity of the requester from the plaintext location information.

\section{Conclusion}

In this paper, we analyzed the threat of unauthorized tracking by compromised RFID Discovery Service in the current industrial standard, EPCglobal Network. To mitigate this threat, we proposed an anti-tracking design in RFID Discovery Service architecture that provides practical privacy protection against unauthorized tracking. Our design protects against database reading attack by a semitrusted Discovery Service and provides efficient key management and access control for dynamic supply chain systems. The design is also compatible with existing communication protocols and database schemas for RFID Discovery Service; therefore, it is practical to integrate our design with the existing systems. Since the specifications of RFID Discovery Service in the EPCglobal Network are still evolving, we hope that our work will contribute in one way or another to the privacy aspect of the ongoing design of RFID Discovery Service.

\section{Acknowledgements}

This work is supported by the Office of Research at Singapore Management University.

\section{References}

[1] S. A. Weis, S. E. Sarma, R. L. Rivest, and D. W. Engels (2004) "Security and privacy aspects of low-cost radio frequency identification systems," in Security in Pervasive Computing, pp. 201-212.

[2] D. Henrici and P. Müller (2004) "Hash-based enhancement of location privacy for radio-frequency identification devices using varying identifiers," in Proceedings of the Second IEEE Annual Conference on Pervasive Computing and Communications Workshops, $\mathrm{p}$. 149.

[3] A. Juels, P. Syverson, and D. Bailey (2005) "Highpower proxies for enhancing rfid privacy and utility," in Proceedings of the 5th Workshop on Privacy Enhancing Technologies.

[4] EPCglobal architecture, http://www.epcglobalinc.org.

[5] EPC Information Services (EPCIS) Version 1.0.1, http://www.epcglobalinc.org/standards/epcis.

[6] B. Fabian, O. Günther, and S. Spiekermann (2005) "Security analysis of the object name service," in International Workshop on Security, Privacy and Trust.

[7] S. Beier, T. Grandison, K. Kailing, and R. Rantzau (2006) "Discovery services-enabling rfid traceability in epcglobal networks," in International Conference on Management of Data.

[8] T. Burbridge and M. Harrison (2009) "Security considerations in the design and peering of rfid discovery services," in Proceedings of IEEE International Conference on RFID, pp. 249-256.

[9] B. Fung, K. Al-Hussaeni, and M. Cao (2009) "Preserving rfid data privacy," in Proceedings of IEEE International Conference on RFID, pp. 200-207.

[10] EPCglobal Object Name Service (ONS) Version 1.0.1, http://www.epcglobalinc.org/standards/ons.

[11] M. Langheinrich (2009) "A survey of RFID privacy approaches," Personal Ubiquitous Comput., vol. 13, no. 6, pp. 413-421. 
[12] R. S. Sandhu (1988) "Cryptographic implementation of a tree hierarchy for access control," Inf. Process. Lett., vol. 27, no. 2, pp. 95-98.

[13] R. Liu and A. Kumar (2003) "Leveraging information sharing to increase supply chain configurability," in Proceedings of the Twenty-Fourth International Conference on Information Systems, pp. 523-536.

[14] M. Blaze, G. Bleumer, and M. Strauss (1998) "Divertible protocols and atomic proxy cryptography," in Proceedings of Eurocrypt '98, LNCS 1403, pp. 127-144.

[15] G. Ateniese, K. Fu, M. Green, and S. Hohenberger (2005) "Improved proxy re-encryption schemes with applications to secure distributed storage," in Proceedings of the 12th Annual Network and Distributed System Security Symposium, pp. 29-43.

[16] JHU-MIT Proxy Re-cryptography Library, http://spar.isi.jhu.edu/prl/

[17] B. Chor, O. Goldreich, E. Kushilevitz, and M. Sudan (1995) "Private information retrieval," in Proceedings of the 36th Annual Symposium on Foundations of Computer Science, pp. 41-50.

[18] D. Chaum (1985) "Security without identification: transaction systems to make big brother obsolete," Commun. ACM, vol. 28, no. 10, pp. 1030-1044.

[19] R. Dingledine, N. Mathewson, and P. Syverson (2004) "Tor: The second-generation onion router," in Proceedings of the 13th USENIX Security Symposium, pp. 303-320. 